\title{
Variables Influencing the Case Prioritisation of Men Convicted of Internet Child Abuse Material Offences
}

\author{
Catherine Garrington ${ }^{a}$, Hannah Merdian ${ }^{\mathrm{b}}$, Douglas P. Boer ${ }^{\mathrm{a}}$ \\ [a] Faculty of Health (Discipline of Psychology), University of Canberra, Canberra, Australia. [b] School of Psychology, \\ University of Lincoln, Lincoln, United Kingdom.
}

Sexual Offending: Theory, Research, and Prevention, 2020, Vol. 15(1), Article e3065,

https://doi.org/10.5964/sotrap.3065

Received: 2019-10-07 • Accepted: 2020-03-18 • Published (VoR): 2020-07-22

Handling Editor: Mark E. Olver, University of Saskatchewan, Saskatoon, SK, Canada

Corresponding Author: Catherine Garrington, Faculty of Health, University of Canberra, ACT 2601 Australia. Email: catherine.garrington@uni.canberra.edu.au

\begin{abstract}
The differentiation between men convicted of child abuse material offences, known as Internet Child Abuse Material (I/CAM), and Contact (CO) child sex offenders is an ongoing area of research. Current research indicates there are differences between men convicted of I/CAM and CO offences. This article highlights key variables that may indicate clinically and/or statistically significant differences between I/CAM and CO populations, as identified in current published research. Identified key variables may contribute to the case prioritisation of men under investigation for I/CAM offences to assist timely investigation. Post conviction, key variables may contribute to targeted treatment, reducing recidivism and protecting the global community. Articles containing variables differentiating I/CAM and CO populations were reviewed; articles that did not include any comparative analysis were excluded. The final sample of articles $(n=10)$ was reviewed using a literature review methodology to collate trend variables and directionality between the two populations. Demographic variables with critical differences between men convicted of I/CAM offences when compared to $\mathrm{CO}$ offences are younger age, White ethnic background, employed, lower use of alcohol and drugs, and less or no recorded criminal history but higher self-reported history of offending. Key psychological variables identified are lower impression management, lower demonstrations of antisociality, higher sexual deviancy and higher levels of victim empathy when compared to men convicted of $\mathrm{CO}$ offences. The findings will be considered in line with existing risk assessment and case prioritisation tools. This research can contribute to community safety through a specific focus on prioritising the investigation, case management, and treatment of men convicted of I/CAM offences and signals future pathways for targeted risk assessment.
\end{abstract}

\section{Keywords}

internet offenders, case prioritisation, child abuse material, risk variables, recidivism 


\section{Highlights}

- Sexual abuse offences against children can be committed online or in person.

- Risk variables differ between Internet Child Abuse Material (I/CAM) and Contact (CO) offence cohorts

- Trend variables and directionality were collated and compared using a literature review methodology

- Key variables may contribute to case prioritisation and timely investigation of I/CAM offences

\section{Offending Pathways}

Research into individuals who use pre-existing Internet Child Abuse Material (I/CAM offenders) online has posited their offence pathways may differ to that of Contact (CO) offenders (see Krone, 2004; Merdian et al., 2014). In contrast to men convicted of I/CAM offenders who commit online offences exclusively, men convicted of CO commit contact sexual offences against children. This may include taking images and/or videos of children to produce child abuse material. I/CAM and CO populations may be distinct; however, some men commit dual offences-both online and contact child sexual offences (Bourke \& Hernandez, 2009). Consideration has been given as to whether men convicted of I/CAM offences progress to $\mathrm{CO}$ offences or vice versa. At this time, there appear to be both similarities and differences between the I/CAM and CO populations and research remains ongoing in this field (see Babchishin et al., 2015).

Research has proposed separating men convicted of I/CAM offences into separate typologies based upon their interactions with child abuse material (e.g., Krone, 2004). Although typologies provide guidance in the types of involvement an individual has with the child abuse material and environment, they do not consider individual variables. Therefore, this paper aims to consider key variables that differentiate between the two main I/CAM and CO populations. For clarity, I/CAM offences may include child abuse material possessors, viewers and downloaders, and $\mathrm{CO}$ offences may include child abuse material producers. An individual, generally a male ${ }^{1}$, may commit one or more of these I/CAM and CO offence types. Both men convicted of I/CAM and CO offences can be exchangers and distributors of child abuse material, noting I/CAM would use pre-existing images where $\mathrm{CO}$ would take new images of children for this purpose.

Riegel (2004) suggested official detection rates of men who use I/CAM may mask self-reported information, with only $6.2 \%$ of his anonymous online survey sample admitting use of CAM which had been detected by authorities. Due to mandatory child abuse reporting laws in many countries, it is difficult to obtain data on offending pathways

1) There is limited research into women who commit online child abuse material offences. All studies included in this paper refer exclusively to men. 
from men undetected by law enforcement. A study comparing undetected and detected $\mathrm{CO}$ and I/CAM users within and between groups found similarities between the samples, however, noted undetected users had higher social functioning and higher sexual selfregulation problems, including sexual preoccupation (Neutze et al., 2012). It is unknown how official detection affects offending pathways.

\section{Key Variables and Case Prioritisation}

\section{Risk Assessment and Sexual Offences}

There has been extensive research into the variables that contribute to sexual reoffending. The development of various risk assessment tools, including the original Static-99 and subsequent versions (Harris, Phenix, Hanson, \& Thornton, 2003), the STABLE-2007 (Hanson, Harris, Scott, \& Helmus, 2007), the Sexual Violence Risk-20 (SVR-20; Boer et al., 1997) and the Violence Risk Scale-Sexual Offense version (VRS-SO; Wong et al., 2003), provide good predictive accuracy in assessing risk of reoffending (Sowden \& Olver, 2016; Rettenberger et al., 2011). Existing risk assessment tools include general static factors (age, prior criminal history), history of violence (past and present) and victim demographics (gender, nature of relationship). Common dynamic risk factors include attitudinal (positive/negative attitudes towards women and/or children), capacity to maintain a significant relationship (past/present) and sexual (deviance, preoccupation, sex as coping). Dynamic risk factors are indicative of areas a man convicted of I/CAM offences may seek to meet needs through offending behaviours and thus important to identify as areas for treatment. In general, these tools apply a cumulative approach to the presence of static and/or dynamic risk variables, and result in a risk of sexual recidivism assessment, generally categorised into low/medium/high or similar, for each individual (Heffernan \& Ward, 2015).

Notably, the above tools are yet to be validated for use exclusively with the I/CAM population, although the Static-99 may be scored on men with prior conviction/s for contact sexual offences who are subsequently convicted of I/CAM offences (Phenix et al., 2016). Early evidence indicates the dynamic risk properties assessed in the STABLE-2007 are applicable to men convicted of I/CAM exclusive of other sexual and general criminal behaviours (Brankley et al., 2019).

\section{Risk Assessment and I/CAM}

Specific to the I/CAM population, there is only one promising risk of re offending tool, the Child Pornography Offender Risk Tool (CPORT), is a tool specifically developed for the risk assessment of men over the age of 18 years convicted of CAM offence/s (Seto \& Eke, 2015). Including three static items and four items categorising the content of CAM found in the offender's possession, the cumulative nature of the CPORT scoring results in higher scores (minimum score of 0 , maximum score of 7) indicating a higher level of projected risk of recidivism over a period of five years. 
In a follow up study, Eke et al. (2019) found the CPORT predicted sexual recidivism (any) $($ AUC $=.72)$ and CAM recidivism $($ AUC $=.74)$ for samples both with and without contact $(\mathrm{CO})$ sexual offences. Additionally, early research indicates the CPORT has good convergent validity with the VRS-SO (Maltais \& Sribney, 2018).

\section{Case Prioritisation}

Law enforcement and offender supervision agencies continually receive new clients and information. The sheer scale of data and files would be unmanageable without successful methods to prioritise information and client file management (Australian Federal Police, 2016). It is critical to ensure the appropriate resources are allocated in a timely manner to ensure community protection remains the priority in investigation and post conviction treatment.

In Australia, the Australian Federal Police (AFP) use a generic Case Categorisation and Prioritisation Model (CCPM) to provide a transparent, objective and consistent basis for managing operational matters (AFP, 2016). The model considers four aspects including the type of matter, the impact on Australian society, the importance of the matter to the client and the AFP, and the resources required to address the matter. The CCPM is a classic example of a generic case prioritisation tool and is applied to all matters reported to the AFP, including reports of online and contact child sexual abuse (AFP, 2016).

Specific to I/CAM populations, the Kent Internet Risk Assessment Tool (KIRAT and KIRAT-2) is a police force work load priority assessment tool (Long et al., 2016). Developed in the United Kingdom by the Kent policing area, the KIRAT and KIRAT-2 assist police to prioritise investigations using a step wise, pathway model based on risk factors for contact sexual offending, in conjunction with professional judgement. Initial research by the developers found the tool to be easy to use, requiring little training and successful in allocating alleged offenders to high risk (very high/high) and low risk (medium/low) priority levels (AUC $=.894$; Long et al., 2016). There has been no further publicly available data since this publication.

General police workload priority tools such as the CCPM, and specific tools such as the KIRAT, clearly contribute to the protection of society by prioritising policing resources. The CCPM provides overarching guidance for case prioritisation for all police matters, while the KIRAT provides case prioritisation within the I/CAM case load. This differentiation enables case prioritisation of the most immediate I/CAM cases. Post investigation and court, case prioritisation and identification of key variables to ensure targeted supervision and treatment. There remains an identified need for tools to identify key variables of clinical relevance that contribute to the risk assessment and treatment specific to I/CAM populations.

It is hypothesised the I/CAM population differs from the CO population. It is further hypothesised the cumulative effect of clinically relevant variables escalates the need for case prioritisation. Noting the ongoing challenges raised above, this paper aims to 
summarise variables identified to date differentiating between the I/CAM and CO populations and may assist in prioritising the investigation, case management and treatment of these specific populations.

\section{Current Research}

This paper extends the systematic review published in Garrington et al. (2018), identifying clinically relevant variables with differences between I/CAM and CO populations. Key findings were mapped against the empirically supported items of the KIRAT case prioritisation and CPORT risk assessment tools. This refinement aims to hone the previous systematic review and clearly detail the variables that differ between the two populations, to provide cause for case prioritisation based on the cumulative effect of these variables. This will continue to add clarity and value to the research into men who commit I/CAM offences and provide direction for case prioritisation and key areas for treatment.

\section{Method}

\section{Search Process}

Articles $(n=17)$ identified in Garrington et al. (2018) were reviewed for methodology, analysis and meaningful effect size differences between the I/CAM and CO populations.

\section{Inclusion Criteria}

Articles included detailed variables related to men convicted of I/CAM offences' risk of reoffending when compared to men convicted of $\mathrm{CO}$ or both I/CAM and CO offences. Articles removed:

- included no statistical analysis (for example, case studies); and/or

- contained analysis with no results with meaningful effect sizes; and/or

- compared I/CAM offenders to general populations; and/or

- were included in meta analyses included in this paper.

The final sample, after exclusions, consisted of 10 articles. See Table 1 for a list of the articles included in the final sample. 


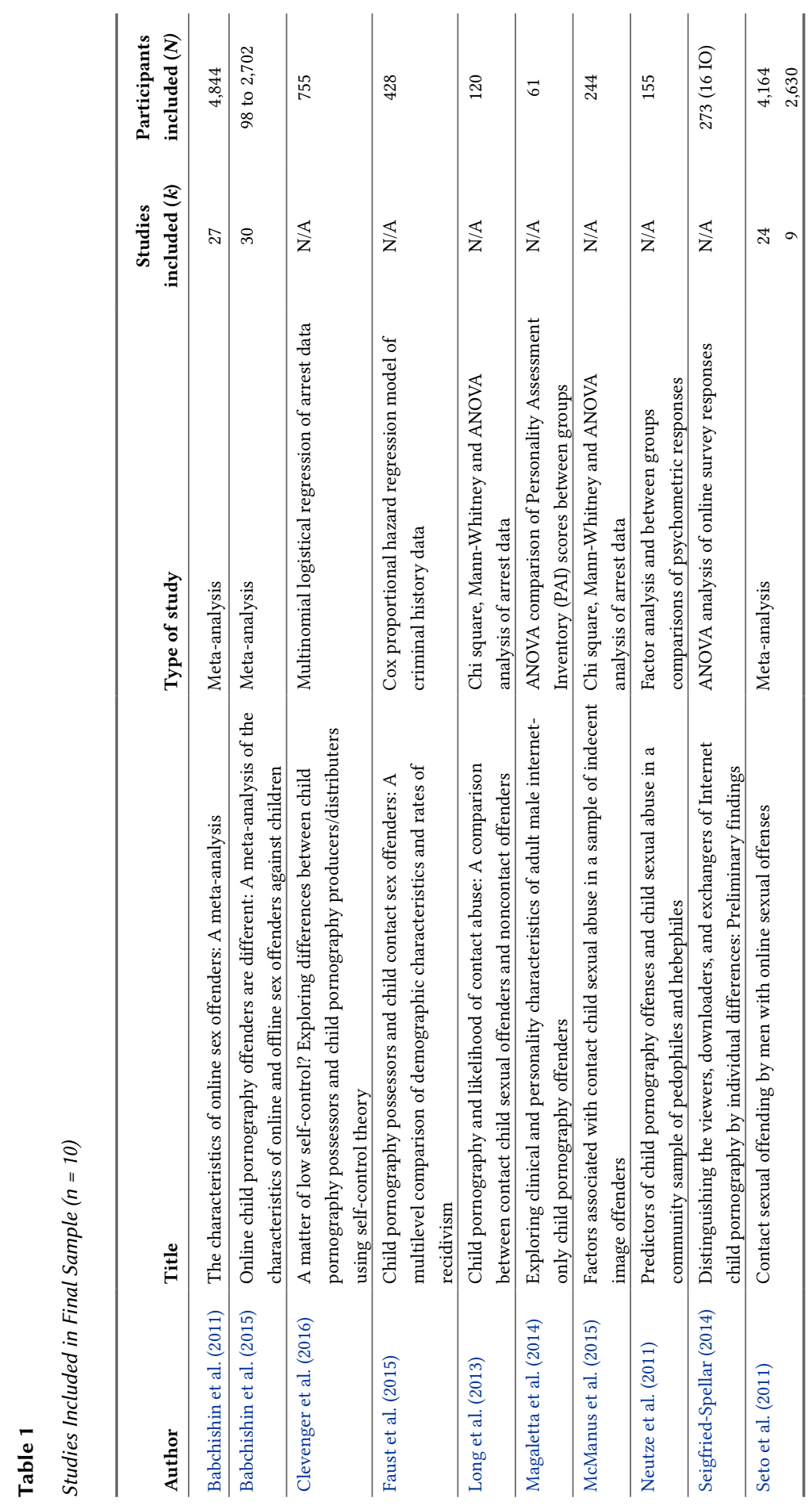

Sexual Offending: Theory, Research, and Prevention 


\section{Results and Discussion}

Babchishin et al. $(2011,2015)$ each completed meta-analyses of characteristics of I/CAM populations, noting the 2014 study is an update of the 2011 study. The 2011 meta-analysis included 27 studies and the 2014 meta-analysis included 30 studies. There are four samples, totalling seven studies, included in both meta analyses. These studies contribute approximately $22 \%$ of the I/CAM data in the 2014 meta-analysis. The 2014 meta-analysis included additional variables when compared to the 2011 meta-analysis. From the published data, it is not possible to identify which studies contribute to each identified variable. Variables included in both meta analyses will not be reported in this paper to avoid reporting the same data twice. Variables unique to each meta-analysis will be reported. We use Cohen's (1988) effect sizes when reporting and interpreting magnitude of effect: small, $d=0.2$; medium, $d=0.5$; and large, $d=0.8$.

\section{Demographic Variables}

\section{Age}

In a meta-analysis of 27 studies $(N=4,844)$, Babchishin et al. (2011) found men convicted of I/CAM offences $(n=1,845)$ were younger than men convicted of CO offences $(n=$ $840), 38.9$ years to 43.6 years in a fixed effect analysis with a small effect size ( $d=$ -0.024). Neutze et al. (2011) concurred in their own study, finding the I/CAM sample was younger, 35.52 years compared to 45.23 years for the CO sample $(d=0.23)$. In contrast, in their own study ( $n=428)$, Faust et al. (2015) found the I/CAM sample to be older than the CO sample, 33.7 years to 22.7 years $(d=0.14)$. The difference in methodologies and sample size may contribute to these findings.

\section{Ethnic Background}

In the same two studies detailed above, Babchishin et al. (2011) and Faust et al. (2015) recorded I/CAM samples to be overwhelmingly, and statistically significantly, White. Babchishin et al. (2011) recorded $8.2 \%$ of the I/CAM sample and $5.4 \%$ of the CO sample to be from racial minority backgrounds. With similar findings, Faust et al. (2015) detailed $93 \%$ of sampled I/CAM offenders and $20.5 \%$ of CO offenders coded as 'White'. Although it is likely there are numerous reasons for these findings, including due to rates of internet access, criminal offence detection and/or conviction rates in different countries, the statistical significance cannot be denied.

\section{Education}

One study retained included a difference in demographic results for years of education. Faust et al. (2015) found $13.7 \%$ of the I/CAM sample were educated for more than 13 years, compared to $11.2 \%$ of the CO sample. Although only one study recorded signifi- 
cant findings, this variable could provide further insights into the typologies of men convicted of I/CAM offences (Krone, 2004).

\section{Employment}

Faust et al. (2015) and Neutze et al. (2011) both established their I/CAM samples to be more likely employed than the CO sample. Specifically, Faust et al. (2015) found $85.8 \%$ of the I/CAM sample were employed compared to $61 \%$ of the CO sample $(p \leq .05)$. Similarly, Neutze et al. (2011) found $79.7 \%$ of their I/CAM sample were employed compared to $49 \%$ of the CO sample and this difference was significant, $\chi^{2}(2, N=115)=12.0, p \leq .01$. Neutze et al. (2011)'s sample of men convicted of I/CAM offences were coded as 'recent offenders', explained as those who admitted use of CAM within the past six months. Although data is limited to two studies, both present similar conclusions.

\section{Living Status}

McManus et al. (2015) provided a detailed breakdown of men convicted of I/CAM offences and their living arrangements when compared to men who commit both I/CAM and $\mathrm{CO}$ offences. Their study reported men who commit both I/CAM and CO offences were more likely to live with either their own children, or a partner and their children than the I/CAM sample $(p<.01) .9 .5 \%(p<.001)$ of men who lived with a partner and their partner's children were identified as men convicted of I/CAM offences (McManus et al., 2015). Similarly, Babchishin et al. (2015) found $16.7 \%$ of men who commit both I/CAM and CO offences were married and/or separated compared to $1.7 \%$ of the I/CAM sample $(p<.05)$. Thus, men who commit I/CAM and CO offences are less likely to be cohabitating with their children, a partner and their children or be married and/or separated than dual offenders. Although these studies do not compare men convicted exclusively of I/CAM offences to men convicted exclusively of $\mathrm{CO}$ offences, rather to men convicted of both offence types, data has been included as it provides insights into the living status of men convicted of I/CAM offences.

\section{Alcohol and Drug Use}

Three studies reported statistically significant results in relation to alcohol and other drug use by a I/CAM sample when compared to a CO sample. Findings varied, with Faust et al. (2015) and Magaletta et al. (2014) both finding their I/CAM sample to have lower use of alcohol and illicit drugs than the CO sample. Specifically, Faust et al. (2015) recorded $13.7 \%$ of men convicted exclusively of I/CAM offences compared to $43.1 \%$ of men convicted exclusively of $\mathrm{CO}$ offences self-reported prior substance use $(p<.01)$.

Using the applicable sub scales of Alcohol Problems (ALC) and Drug Problems (DRG) from the Personality Assessment Inventory (PAI), Magaletta et al. (2014) found an I/CAM sample reported lower excessive use than the CO sample for both alcohol, $F(1,61)=6.74$, $p<.01$, and drugs, $F(1,61)=7.98, p<.001$. Men convicted of I/CAM offences recorded 
scores below and men convicted of $\mathrm{CO}$ offences recorded scores above the mean for male normative samples.

In comparison, Clevenger et al. (2016) recorded 51\% of an I/CAM sample self-reported higher use of alcohol and other drugs at the time of the crime compared to $34.4 \%$ of the CO sample. This difference was significant, $\chi^{2}(2, n=755)=23.52, p \leq .01$.

The three studies reported were based on the researchers' own data with samples sizes of $N=61$ (Magaletta et al., 2014), $N=428$ (Faust et al., 2015), and $N=755$ (Clevenger et al., 2016). These findings indicate further research is required, although early indications are that use of alcohol and other drugs may be a contributing factor to reoffending. It is noted alcohol and other drug use is strongly correlated to general re offending and contributes to the reduction of inhibitions (Dowden \& Brown, 2002).

\section{Victim of Past Physical/Sexual Abuse}

With regard to sexual offenders being the victim of past physical and/or sexual abuse, one study reported statistically significant findings. Faust et al. (2015) found $11.7 \%$ of men convicted of I/CAM offences compared to $17.6 \%$ of men convicted of CO offences sampled reported being the victim of past physical and/or sexual abuse $(p \leq .05)$. Babchishin et al. (2011) found men convicted of I/CAM offences also reported lower levels of past physical abuse than men convicted of CO offences $(24.4 \%$ and $40.8 \%$ respectively), significant in a fixed effect analysis. They found no difference in reporting rates of past sexual abuse between I/CAM and CO offenders. Again, conclusions cannot be drawn from one study, and further research is required.

For general comparison, research places the prevalence of child sexual abuse in the United States of America (USA), the same location as the above research, as approximately $8.3 \%$ (Finkelhor et al., 2005). This indicates both men who commit I/CAM and/or CO offences may have been subject to higher levels of child sexual abuse than the general population.

\section{Criminal History (General and Sexual) - Recorded}

Three studies were found to contain statistically significant results in relation to recorded criminal histories (general and sexual) for I/CAM, CO and dual samples. Clevenger et al. (2016) recorded $61.1 \%$ of men convicted of I/CAM offences compared to $29.0 \%$ men convicted of $\mathrm{CO}$ offences sampled had less and/or no prior recorded arrests for sexual offences and this difference was significant, $\chi^{2}(2,755)=9.29, p \leq .01$. Faust et al. (2015) concurred, finding $58 \%$ of an I/CAM sample compared to $25.2 \%$ of a CO sample had less and/or no recorded criminal history $(p \leq .05)$. However, Long et al. (2013) reported different findings, with $21.7 \%$ of an I/CAM sample compared to $58.3 \%$ of dual offence type sample had less and/or no prior recorded criminal history $(p<.001)$. Long et al.'s (2013) study compared an I/CAM sample to a dual I/CAM and CO sample rather than an 
exclusive $\mathrm{CO}$ sample. These findings indicate there may be further differences between the I/CAM, CO and dual offence type populations that have yet to be quantified.

\section{Criminal History (Sexual Only) - Self Reported}

Neutze et al. (2011) reported men recently convicted of I/CAM offences self-reported prior, undetected, I/CAM, CO and/or dual offences at a rate of $87.5 \%$, and an inactive I/CAM sample at 77.5\%. "Inactive offenders" were defined as those who reported no further offences in the preceding six-month period. When compared to the self-reported re offence rate of a CO sample at $98 \%$, the results were significant $(p<.01)$. Approximately $11 \%$ of the original sample $(n=155)$ was excluded from the reported analysis due to missing data. Whilst noting the statistical significance of this finding, the high level of self-report is also noted.

Seto et al.'s (2011) meta-analysis of 21 studies found approximately $12 \%$ of an I/CAM sample had a recorded criminal history for contact sexual offending. However, approximately $55 \%$ of the I/CAM sample self-reported contact sexual offending and this proportion was significant $(p<.001)$. Both studies indicate the proportion of I/CAM populations with prior undetected offences is significantly higher than recorded on official criminal histories.

\section{Psychological Variables}

\section{Social/Inter Personal Deficits}

Returning to Magaletta et al.'s (2014) study using the PAI, they found men convicted of I/CAM offences recorded less desire for control than the CO sample, $F(2,61)=4.78$, $p<.001$, when using the Dominance Interpersonal Scale. I/CAM scores $(M=47)$ were below, and CO scores $(M=54)$ were above the male normative scores $(M=52)$ on the PAI. Again, noting one study cannot draw definitive conclusions, this adds further direction to the possible risk variables for men convicted of I/CAM offences.

\section{Impression Management}

Men convicted of I/CAM offences were found to engage in less socially desirable responding (impression management) than men convicted of $\mathrm{CO}$ offences in a study by Babchishin et al. (2011). This result was small to moderate in a fixed effect analysis ( $d=-0.038)$, using meta-analysis data collated from studies $(k=27)$ using Paulhus's Impression Management Scale (IMS; Paulhus, 1998) and an earlier version in German (Paulhus, 1991). Although these results are from one meta-analysis, the large total sample size $(N=4,844)$ is noted. 


\section{Impulse Seeking}

Seigfried-Spellar (2014) found a lower level I/CAM sample to have lower levels of impulse seeking behaviours than a higher level I/CAM sample $(p<.10)$. Whilst it is again noted conclusions cannot be drawn from one study, and there is no comparison to men convicted exclusively of $\mathrm{CO}$ offences, this provides an interesting area for further research in relation to I/CAM populations.

\section{Antisociality}

Returning to the use of the PAI, Magaletta et al. (2014) found an I/CAM sample scored lower on the Antisociality subscale than a CO sample, $F(2,61)=10.58, p<.001$. In comparison to the mean for male normative samples $(M=53)$, the I/CAM sample recorded scores $(M=54)$ close to the mean and the CO sample recorded scores $(M=63)$ well above.

Babchishin et al.'s (2015) meta-analysis of 30 studies found antisociality to be a higher correlating risk factor $(p<.001)$ fora CO sample than an I/CAM sample. The term 'antisociality' in Babchishin et al.'s (2015) meta-analysis comprised results from higher numbers of prior offences, higher scores on antisociality measures and greater problems with supervision than a CO sample. Data from Magaletta et al. (2014) is included in Babchishin et al. (2015). Both results are presented due to the addition of extra data in Babchishin et al. (2015), but necessarily must be interpreted with caution. These results indicate men convicted of I/CAM offences are less likely to demonstrate antisocial tendencies than men convicted of $\mathrm{CO}$ offences.

\section{Offence Specific Variables}

The Babchishin et al. (2011, 2015) meta-analyses identified several offence specific variables differentiating I/CAM and CO men, reviewed as follows.

\section{Access to Internet}

Babchishin et al. (2015) established an I/CAM sample to have higher access to the internet than a CO sample when indicated by younger age $(d=-0.21)$, higher income $(d=$ $0.60)$ and higher education $(d=0.77)$ in small, moderate and moderate to high effect sizes respectively, in fixed effect analyses.

\section{Access to Children/Opportunity}

In Babchishin et al. (2015), an I/CAM sample were found to have moderate to lower access to children than a CO sample $(d=0.32)$ in a fixed effect analysis. To determine whether this is a coincidental or causative variable requires further research. 


\section{Sexual Deviance}

Babchishin et al. (2011) reported an I/CAM sample to have moderate incidences of sexual deviance than a CO sample $(d=-0.57)$. In their 2014 updated meta-analysis, Babchishin et al further confirmed an I/CAM sample to demonstrate moderate and low moderate levels respectively of sexual deviancy when compared to a CO sample, coded as pedohebephilia (any paraphilia) $(d=0.50)$ and pedophilia $(d=0.37)$ in random effect analyses. Babchishin et al.'s (2015) meta-analysis includes data from their 2011 meta-analysis with additional studies and must be interpreted with caution. These findings posit questions as to whether men convicted of I/CAM offences have more self-control than men who commit $\mathrm{CO}$ offences and therefore do not act on their deviant impulses.

\section{Victim Empathy}

Babchishin et al. (2011) further found an I/CAM sample to have higher levels of victim empathy when compared to a CO sample $(d=0.56)$. They further confirmed this finding in their later meta-analysis $(d=0.53$; Babchishin et al., 2015) with the inclusion of additional studies. Again, this moderate variable could be of further interest in I/CAM research when considered with other psychological variables, including sexual deviance.

\section{Summary}

Key variables have been identified in selected published research that may contribute to the case prioritisation and risk assessment of individuals who use I/CAM. The identified key variables were then mapped against the CPORT and KIRAT empirically supported items. Please see Table 2 for a comparison between the identified key variables and the CPORT and KIRAT items. 


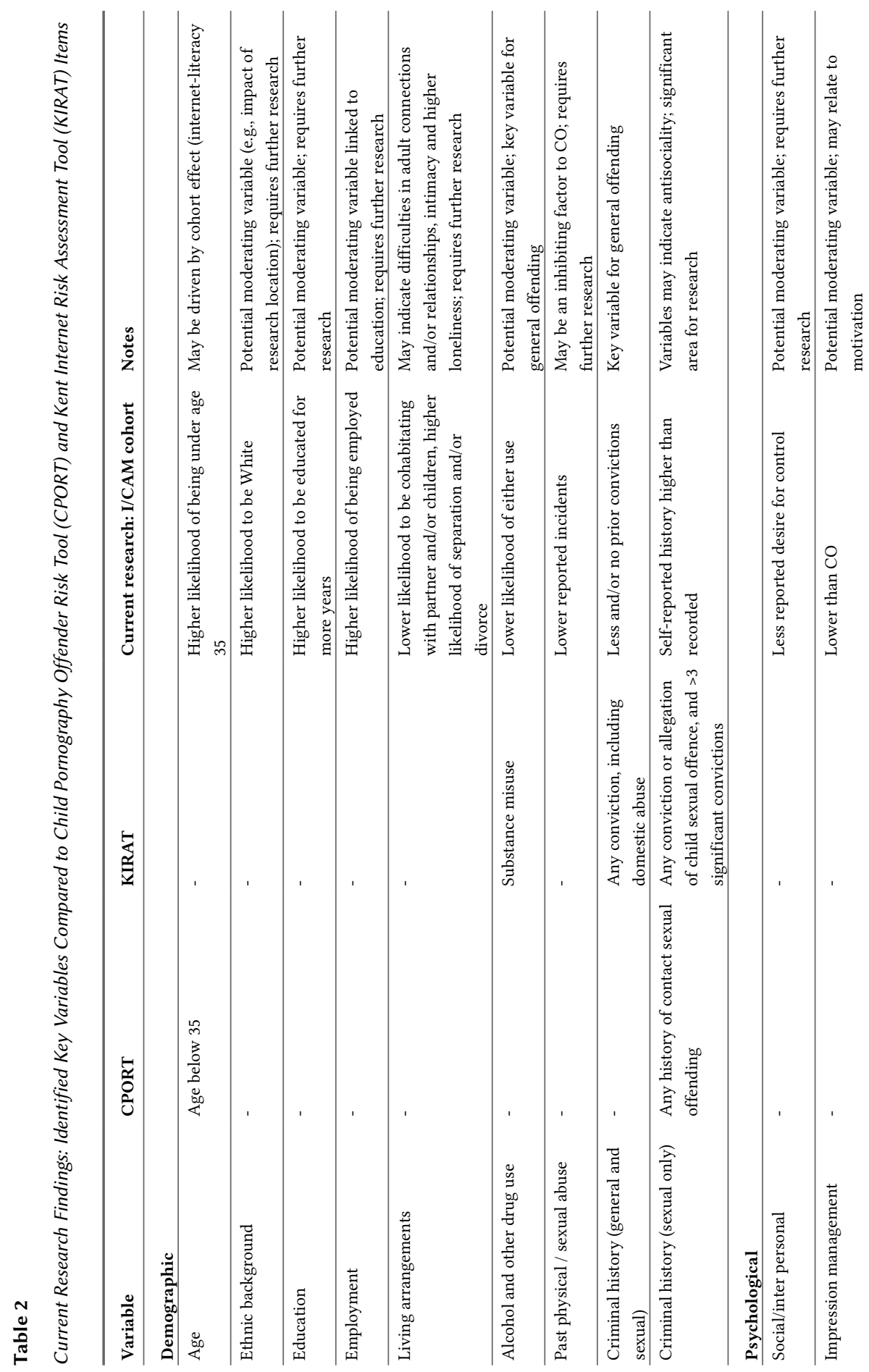

Sexual Offending: Theory, Research, and Prevention 


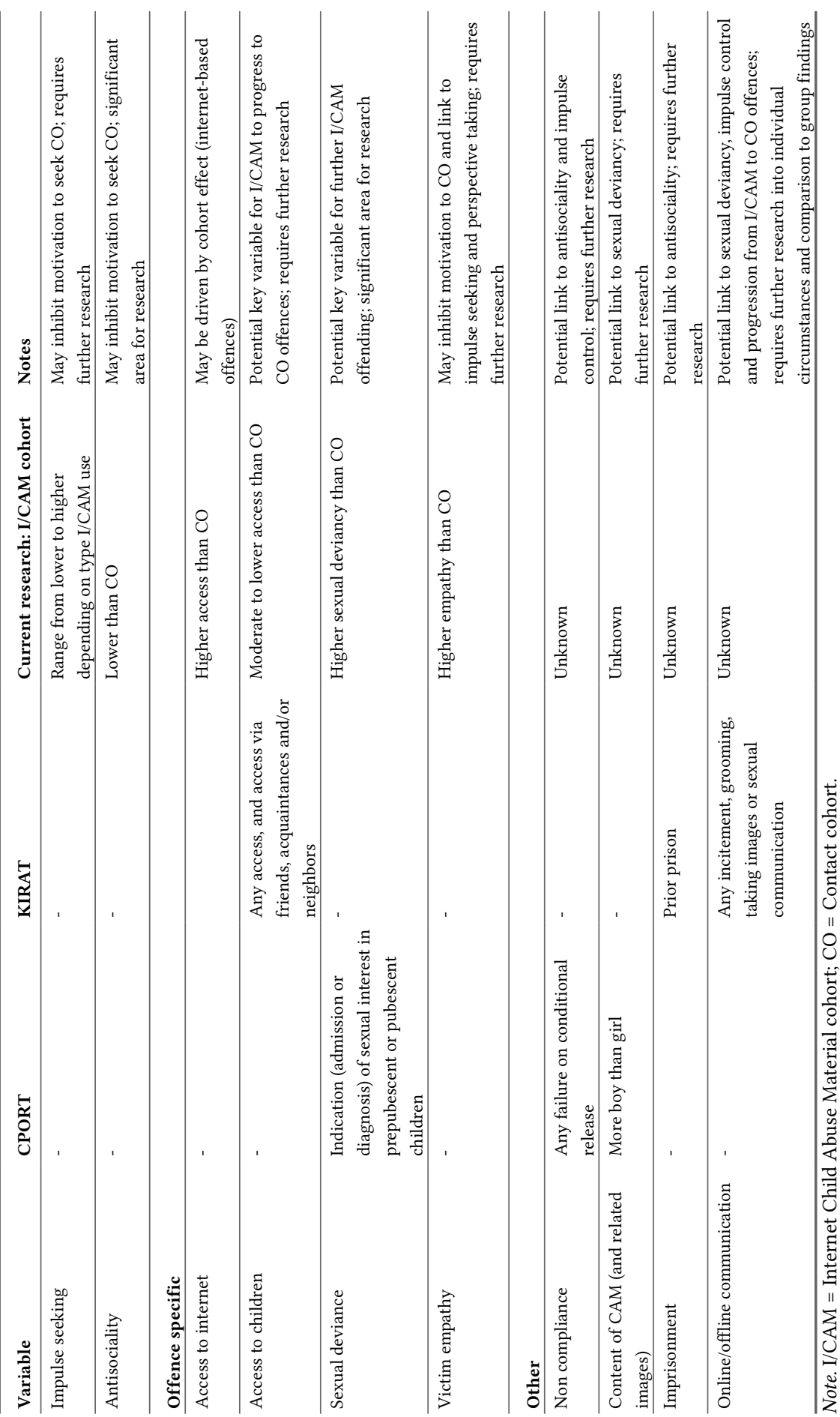




\section{Conclusion}

Extending the systematic review published in Garrington et al. (2018), this research reviewed relevant publications that identify differences between I/CAM and CO populations. Identified clinically significant, or key, variables were mapped against the empirically supported items of the KIRAT case prioritisation and CPORT risk assessment tools to establish areas for future direction in case prioritisation and treatment.

Considering the identified key variables in a case prioritisation context, it is likely the cumulative effect of identified variables could result in a higher risk of continued offending behaviour prior to arrest, or prior to an individual's awareness of detection. Thus, the case prioritisation for investigation by police could result in reduced time from detection to arrest, focussed investigations and improvement of community protection. This could limit an individual's opportunities and timeframe to continue I/CAM offending and reduce the victimization of children online. This is consistent with the principles of the KIRAT, including focussing resources in case prioritisation to ensure investigations are conducted in a timely manner commensurate with assessed prioritisation levels.

In a post detection context, case prioritisation based on clinically relevant variables could be incorporated into monitoring to offer focus to supervision and interventions such as those offered by correctional services. The early results of the CPORT are promising in providing I/CAM cohort specific risk assessment and informing treatment targets.

Given the plethora of research in recidivism of men convicted of general sex offences, it is important to consider the differences above as early indications that men who commit I/CAM offences present unique characteristics This concurs with the decisions by the respective authors of the STATIC-99 (Harris, Phenix, Hanson, \& Thornton, 2003) and subsequent versions, the STABLE-2007 (Hanson et al., 2007) and the SVR-20 (Boer et al., 1997), in not applying standard sexual offender risk assessment tools in isolation and in the absence of further research, to the I/CAM cohort. The recognition that case prioritisation informs police investigations, supervision by corrective services, and treatment needs is key to ensuring systemic linkages in offender management. Whilst there is promising research in this area, it is expected the coming years will result in modified and/or further scoring guidelines for both case prioritisation and risk assessment tools.

\section{Limitations}

This paper recognises the inherent limitations in conducting an in depth follow up study using the articles identified in Garrington et al. (2018). The conclusions regarding risk variables and directionality is noted to be based on a group of studies and may not represent all available published research. Additionally, it is noted statistically significant data for the I/CAM population is, to date, limited. It is likely further research will either strengthen the significance of variables or weaken the predictive value. 
There are recognised ongoing limitations in this field, notably the low numbers of published articles comparing the I/CAM and CO populations. There remain challenges in the comparison of data drawn from a range of methodological bases and caution is advised in interpreting current results.

\section{Further Directions}

Based on the limitations above, a significant research need is ongoing research into the CPORT, and the development of further specific risk assessment tools for this cohort. The comparison between key variables identified in this paper, CPORT and KIRAT items provide the first step in monitoring variables through case prioritisation and risk assessment. The continuity of variable inclusion contributes to shared language from detection, conviction and treatment and provides added scope for further research. New and emerging research, including longitudinal studies and gap analysis will contribute to the clarification of key variables and amendments to case prioritisation and treatment.

Although it remains early days for drawing significant conclusions, indicative data suggests the I/CAM population without a history of contact sex offending differs from the $\mathrm{CO}$ population. This again highlights the importance of focused risk assessment, identification of risk variables and subsequent targeted interventions to reduce recidivism behaviour.

Funding: The authors have no funding to report.

Competing Interests: The authors have declared that no competing interests exist.

Acknowledgments: The authors have no support to report.

\section{References}

Australian Federal Police. (2016). The case categorisation and prioritisation model. Retrieved from https://www.afp.gov.au/sites/default/files/PDF/ccpm-july-2016.pdf

Babchishin, K. M., Hanson, R. K., \& Hermann, C. A. (2011). The characteristics of online sex offenders: A meta-analysis. Sexual Abuse, 23(1), 92-123. https://doi.org/10.1177/1079063210370708

Babchishin, K. M., Hanson, R. K., \& VanZuylen, H. (2015). Online child pornography offenders are different: A meta-analysis of the characteristics of online and offline sex offenders against children. Archives of Sexual Behavior, 44(1), 45-66. https://doi.org/10.1007/s10508-014-0270-x

Boer, D. P., Hart, S. D., Kropp, P., \& Webster, C. (1997). Manual for the Sexual Violence Risk-20. Vancouver, Canada: The British Columbia Institute Against Family Violence. 
Bourke, M. L., \& Hernandez, A. E. (2009). The 'Butner Study' redux: A report of the incidence of hands-on child victimization by child pornography offenders. fournal of Family Violence, 24(3), 183-191. https://doi.org/10.1007/s10896-008-9219-y

Brankley, A. E., Babchishin, K. M., \& Hanson, R. K. (2019). STABLE-2007 demonstrates predictive and incremental validity in assessing risk-relevant propensities for sexual offending: A metaanalysis. Sexual Abuse. Advance online publication. https://doi.org/10.1177/1079063219871572

Clevenger, S. L., Navarro, J. N., \& Jasinski, J. L. (2016). A matter of low self-control? Exploring differences between child pornography possessors and child pornography producers/ distributers using self-control theory. Sexual Abuse, 28(6), 555-571. https://doi.org/10.1177/1079063214557173

Cohen, J. (1988). Statistical power analysis for the behavioral sciences (2nd ed.). Hillsdale, NJ, USA: Lawrence Erlbaum Associates.

Dowden, C., \& Brown, S. (2002). The role of substance abuse factors in predicting recidivism: A meta-analysis. Psychology, Crime \& Law, 8(3), 243-264.

https://doi.org/10.1080/10683160208401818

Eke, A. W., Helmus, L. M., \& Seto, M. C. (2019). A validation study of the Child Pornography Offender Risk Tool (CPORT). Sexual Abuse, 31(4), 456-476. https://doi.org/10.1177/1079063218762434

Faust, E., Bickart, W., Renaud, C., \& Camp, S. (2015). Child pornography possessors and child contact sex offenders: A multilevel comparison of demographic characteristics and rates of recidivism. Sexual Abuse, 27(5), 460-478. https://doi.org/10.1177/1079063214521469

Finkelhor, D., Ormrod, R., Turner, H., \& Hamby, S. (2005). The victimization of children and youth: A comprehensive, national survey. Child Maltreatment, 10(1), 5-25. https://doi.org/10.1177/1077559504271287

Garrington, C., Rickwood, D., Chamberlain, P., \& Boer, D. P. (2018). A systematic review of risk variables for child abuse material offenders. Journal of Forensic Practice, 20(2), 91-101. https://doi.org/10.1108/JFP-05-2017-0013

Hanson, R. K., Harris, A. J. R., Scott, T. L., \& Helmus, L. M. (2007). Assessing the risk of sexual offenders on community supervision: The Dynamic Supervision Project. Ottawa, Canada: Public Safety Canada.

Harris, A., Phenix, A., Hanson, R. K., \& Thornton, D. (2003). STATIC-99 Coding Rules Revised 2003. Retrieved from https://www.researchgate.net/publication/228592243_Static_99_Coding_Rules_Revised_2003

Heffernan, R., \& Ward, T. (2015). The conceptualization of dynamic risk factors in child sex offenders: An agency model. Aggression and Violent Behavior, 24(5), 250-260. https://doi.org/10.1016/j.avb.2015.07.001

Krone, T. (2004). A typology of online child pornography offending (Trends and Issues in Crime and Criminal Justice, No. 279). Canberra, Australia: Australian High Tech Crime Centre. 
Long, M. L., Alison, L. A., \& McManus, M. A. (2013). Child pornography and likelihood of contact abuse: A comparison between contact child sexual offenders and noncontact offenders. Sexual Abuse, 25(4), 370-395. https://doi.org/10.1177/1079063212464398

Long, M., Alison, L., Tejeiro, R., Hendricks, E., \& Giles, S. (2016). KIRAT: Law enforcement's prioritization tool for investigating indent image offenders. Psychology, Public Policy, and Law, 22(1), 12-21. https://doi.org/10.1037/law0000069

Magaletta, P. R., Faust, E., Bickart, W., \& McLearen, A. M. (2014). Exploring clinical and personality characteristics of adult male internet-only child pornography offenders. International fournal of Offender Therapy and Comparative Criminology, 58(2), 137-153.

https://doi.org/10.1177/0306624X12465271

Maltais, N., \& Sribney, C. (2018). Assessing the convergent validity of the VRS-SO and the CPORT with a forensic community sample. Conference presentation at the 37th annual research and treatment conference of the Association of the Treatment of Sexual Abusers, Vancouver, BC, Canada.

McManus, M. A., Long, M. L., Alison, L., \& Almond, L. (2015). Factors associated with contact child sexual abuse in a sample of indecent image offenders. Journal of Sexual Aggression, 21(3), 368-384. https://doi.org/10.1080/13552600.2014.927009

Merdian, H. L., Curtis, C., Thakker, J., Wilson, N., \& Boer, D. P. (2014). The endorsement of cognitive distortions: Comparing child pornography offenders and contact sex offenders. Psychology, Crime \& Law, 20(10), 971-993. https://doi.org/10.1080/1068316X.2014.902454

Neutze, J., Grundmann, D., Scherner, G., \& Beier, K. M. (2012). Undetected and detected child sexual abuse and child pornography offenders. International fournal of Law and Psychiatry, 35(3), 168-175. https://doi.org/10.1016/j.ijlp.2012.02.004

Neutze, J., Seto, M. C., Schaefer, G. A., Mundt, I. A., \& Beier, K. M. (2011). Predictors of child pornography offenses and child sexual abuse in a community sample of pedophiles and hebephiles. Sexual Abuse, 23(2), 212-242. https://doi.org/10.1177/1079063210382043

Paulhus, D. L. (1991). Measurement and control of response bias. In J. P. Robinson, P. R. Shaver, \& L. S. Wrightsman (Eds.), Measures of personality and social psychological attitudes (pp. 17-59). London, United Kingdom: Academic Press.

Paulhus, D. L. (1998). Manual for the Balanced Inventory of Desirable Responding (Version 7). Toronto, Canada: Multi-Health Systems.

Phenix, A., Fernandez, Y., Harris, A. J. R., Helmus, L. M., Hanson, R. K., \& Thornton, D. (2016). STATIC-99R coding rules revised 2016. Retrieved from http://www.static99.org/pdfdocs/Coding_manual_2016_v2.pdf

Rettenberger, M., Boer, D. P., \& Eher, R. (2011). The predictive accuracy of risk factors in the Sexual Violence Risk-20 (SVR-20). Criminal fustice and Behavior, 38(10), 1009-1027. https://doi.org/10.1177/0093854811416908

Riegel, D. L. (2004). Effects on boy-attracted pedosexual males of viewing boy erotica. Archives of Sexual Behavior, 33(4), 321-323. https://doi.org/10.1023/B:ASEB.0000029071.89455.53 
Seigfried-Spellar, K. (2014). Distinguishing the viewers, downloaders, and exchangers of Internet child pornography by individual differences: Preliminary findings. Digital Investigation, 11(4), 252-260. https://doi.org/10.1016/j.diin.2014.07.003

Seto, M. C., \& Eke, A. W. (2015). Predicting recidivism among adult male child pornography offenders: Development of the Child Pornography Offender Risk Tool (CPORT). Law and Human Behavior, 39(4), 416-429. https://doi.org/10.1037/lhb0000128

Seto, M. C., Hanson, R. K., \& Babchishin, K. (2011). Contact sexual offending by men with online sexual offenses. Sexual Abuse, 23(1), 124-145. https://doi.org/10.1177/1079063210369013

Sowden, J. N., \& Olver, M. E. (2016). Use of the Violence Risk Scale-Sexual Offender Version and the Stable 2007 to assess dynamic sexual violence risk in a sample of treated sexual offenders. Psychological Assessment, 29(3), 293-303. https://doi.org/10.1037/pas0000345

Wong, S. P., Olver, M. E., Nicholaichuk, T. P., \& Gordon, A. (2003). The Violence Risk Scale-Sexual Offense version (VRS-SO). Saskatchewan, Canada: Regional Psychiatric Centre and University of Saskatchewan.

Sexual Offending: Theory, Research, and Prevention is the official journal of the International Association for the Treatment of Sexual Offenders (IATSO).

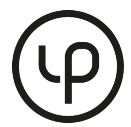

leibniz-psychology.org

PsychOpen GOLD is a publishing service by Leibniz Institute for Psychology Information (ZPID), Germany. 